\title{
Ppkausas, una red digital juvenil que defendió el sistema
}

neoliberal

Ppkausas, a Youth Digital Network that Defended the

Neoliberal System

BRUNO RIVAS FRÍAS

Magíster en Estudios Culturales y bachiller en Ciencias y Artes de la Comunicación con mención en Periodismo por la Pontificia Universidad Católica del Perú (PUCP). Es docente del Departamento de Comunicaciones de la PUCP y del Departamento de Humanidades de la Universidad Peruana de Ciencias Aplicadas. Su investigación analiza las subjetividades que se manifestaron en una red social virtual formada por jóvenes peruanos durante una campaña electoral. Ha publicado sobre las representaciones de la globalización que construyen las industrias de contenido. 



\section{Pplkausas, una red digital juvenil que defendió el sistema neoliberal \\ Ppkausas, a Youth Digital Network that Defended the Neoliberal System}

Bruno Rivas Frías

Pontificia Universidad Católica del Perú / Universidad Peruana de Ciencias Aplicadas bruno.rivas@pucp.edu.pe

Recibido: 12-05-2019 / Aceptado: 28-09-2019

https://doi.org/10.1880o/conexion.201902.005

\section{PALABRAS CLAVE / KEYWORDS}

Redes sociales virtuales, movimientos sociales, juventud, campañas políticas, subjetividades / Virtual social networks, social movements, youth, political campaigns, subjectivities

\section{RESUMEN}

El presente artículo tiene como objetivo analizar la red social virtual que formaron los jóvenes que participaron en la campaña presidencial de Pedro Pablo Kuczynski en el 2011. Para conseguir dicho objetivo, se aplicó una metodología cualitativa que consistió en un trabajo de campo compuesto por entrevistas a profundidad realizadas a 24 miembros del colectivo Generación Joven PPK, los llamados ppkausas. Como producto del análisis, se pudo observar que este movimiento utilizó las herramientas virtuales para generar un espacio que saliera en defensa del sistema hegemónico. Como conclusión, el caso analizado muestra que las redes ju- veniles pueden ser espacios en los que se agrupan individuos cuyas subjetividades han sido marcadas por los discursos del individualismo.

\section{ABSTRACT}

The purpose of this article is to analyze the virtual social network formed by the young people who participated in the presidential campaign of Pedro Pablo Kuczynski in 2011. In order to achieve this objective, a qualitative methodology was applied that consisted of a field work composed of interviews in depth carried out to 24 members of the Young Generation PPK collective, the so-called ppkausas. As a result of the analysis, it was observed that this movement used virtual tools to generate a space that came out in defense of the hegemonic system. In conclusion, the case analyzed shows that youth networks can be spaces in which individuals whose subjectivities have been marked by the discourses of individualism are grouped. 


\section{Ppkausas, una red digital juvenil que defendió el sistema neoliberal ${ }^{1}$}

\section{Introducción: una red social juvenil que participó en política}

Desde la década de los noventa, la figura del joven peruano ha estado asociada a la apatía política (Venturo, 2001; Portocarrero, 2001). Dicho período coincide con el establecimiento del neoliberalismo como sistema político-económico imperante en el país, fenómeno que cambió las subjetividades en la población. La subjetividad hegemónica que se estableció con esa doctrina económica fue la del hombre de éxito, en perjuicio de la del militante político (Portocarrero, 2001). Todo esto ha provocado que, durante más de 25 años, la juventud peruana haya estado construyendo su identidad a partir del discurso del emprendimiento y de la figura del empresario emergente. Siguiendo el mandato de la globalización, el joven peruano se esfuerza por integrarse al mundo del consumo.

Sin embargo, en los últimos años, algunos sucesos ocurridos en el Perú han puesto en cuestión aquel sentido común que indica que los jóvenes no tienen interés en la acción política. Una prueba de ello son las movilizaciones encabezadas por universitarios o jóvenes trabajadores disconformes con la forma en que se ejerce el poder en el país o grupos juveniles haciendo campaña por el candidato que consideraban capaz de revolucionar la nación. Se trata de manifestaciones ocurridas en una época en que un espíritu de revuelta se ha extendido por el mundo cuestionando el sistema establecido por el modelo neoliberal. Dichos colectivos se han apoyado en las herramientas tecnológicas que se han expandido durante las primeras décadas del siglo XXI para realizar convocatorias que permitan tomar las calles.

En este artículo, se analizará el caso de un grupo que aparentemente rompió la apatía política al participar en una campaña electoral a través del uso de herramientas digitales. A partir del caso de los ppkausas, los jóvenes que participaron en la campaña presidencial de Pedro Pablo Kuczynski (PPK) en el 2011, se buscará analizar el uso que un grupo de jóvenes peruanos les dieron a las redes sociales virtuales para defender su postura política. Asimismo, el caso permitirá reconocer que incluso en los grupos que participan en campañas electorales se reproducen las dinámicas de consumo que reducen la política a un proceso efímero y alejado del compromiso.

\section{Marco teórico: las redes juveniles despiertan}

Desde el 2010, el mundo ha sido testigo del surgimiento de movimientos de protesta en diferentes lugares del planeta

${ }^{1}$ Este artículo es producto de la investigación realizada en la tesis de maestría Ppkausas, defensores del nuevo capitalismo peruano. Un estudio del discurso de 24 jóvenes que participaron en la campaña de Pedro Pablo 
que tienen un rasgo en común: el uso de las redes sociales como instrumento de convocatoria para marchas y movilizaciones que buscan plantarle cara a los órganos de poder y generar cambios en las sociedades.

Para poder entender estos movimientos, es necesario definir primero el concepto de redes sociales. Castells indica que una red es «un conjunto de nodos interconectados» (2009, p. 45) por el que circula información. Asimismo, Hansen, Shneiderman y Smith definen las redes sociales como «un conjunto de herramientas virtuales (online tools) que apoyan la interacción entre los usuarios» (citados por León, 2013, p. 249). Por lo tanto, dicho concepto es comúnmente vinculado a las innovaciones tecnológicas que aparecieron al inicio del siglo XXI. Sin embargo, autores como Christakis y Fowler (2010) nos recuerdan que estas estructuras no le son extrañas al ser humano, ya que le han servido para organizarse desde los inicios de las comunidades humanas. Somos parte de una familia, hemos acudido al colegio, a la universidad, trabajamos, entre otros. Cada una de esas experiencias permite que vayamos formando parte de redes sociales en las que somos representados por nodos y en las que generamos vínculos con los demás integrantes. Justamente lo que circula en esa red es lo que la define. Nuestra condición de puntos de contacto de un amplio tramado que permite la difusión de información provoca que dependamos de esas redes y que nuestra subjetividad se vea condicionada por ellas.

Castells (2009) explica que las redes siguen programas que son asignados por los actores sociales que ostentan el poder y a los que califica de programadores. Por ejemplo, si una red vecinal decidiera organizarse para votar de forma unificada en unas elecciones municipales, su decisión conjunta podría definirse por la acción de un actor social que sea visto como una autoridad. Información proveniente de un medio de comunicación o un líder comunitario respetados puede ser determinante para la elección que realice la red en conjunto. La decisión colectiva sería, entonces, consecuencia de una programación planteada por actores sociales con poder. No obstante, hay que aclarar que es posible alcanzar cierto grado de independencia en las redes. Castells indica que «resistirse a la programación e interrumpir las conexiones para defender valores e intereses son las formas de contrapoder que ejercen los movimientos sociales y la sociedad civil» (2009, p. 84). Por lo tanto, es posible crear redes que planteen una programación distinta a la propuesta por los actores sociales. Por ejemplo, la propuesta puede descubrir que la información que se difunde sobre los candidatos es falsa y plantear una campaña en contra. El surgimiento de movimientos sociales de protesta que se organizan a través de las herramientas tecnológicas durante la última década sería una muestra del uso del citado contrapoder. 
Castells (2012) propone que vivimos tiempos de «indignación y esperanza» debido a que la aparición en el 2011 de movimientos sociales, como los indignados del 15-M español u Occupy Wall Street, puso en evidencia que existe un descontento con el statu quo y con la forma como se ejerce el poder en el mundo. Dichos grupos ejercieron el contrapoder, ya que lograron construir un nuevo espacio público al combinar un trabajo en redes sociales con la ocupación de un espacio urbano. Esos movimientos despiertan la esperanza de que se concrete una política insurgente (Castells, 2012) que rompa con los centros de poder político tradicional y plantee cambios en la forma en que se estructuran las redes políticas. El advenimiento de esa política podría provocar que se rompa con los sentidos comunes de la época y que se postulen alternativas frente al sistema económico neoliberal que se ha impuesto en el mundo. Estos grupos aparecen como las barricadas contemporáneas en las que se estacionan los nuevos revolucionarios. Asimismo, Badiou encontró en esos grupos «la posibilidad de la posibilidad» (2012, p. 44) de una revuelta histórica. Badiou (2012) define la revuelta histórica como las movilizaciones que se gestan en un periodo en el que hay indignación contra el sistema imperante. Durante ese tiempo, se plantean alternativas al sistema pero no se gesta una revolución, porque aún no termina de configurarse una propuesta afirmativa capaz de generar un cambio. Siguiendo a ambos posibilidad de un acontecimiento a partir de ejercer un contrapoder que se resista a los sentidos comunes impuestos por los centros hegemónicos.

No obstante, a contraparte de lo expuesto anteriormente, Byung-Chul Han desconfía de estas redes de indignación, porque considera que son efímeras y que están constituidas por sujetos individualistas que solo piensan en su propio beneficio. Para el autor, las redes sociales virtuales representan un enjambre digital formado por individuos aislados que siguen la tendencia neoliberal de la época (2014, p. 22).

Una de las características de estas redes de insurgencia es que la mayoría ha estado integrada por jóvenes. Estos movimientos han ido a contracorriente de la subjetividad hegemónica favorable al mercado que se extendió por el mundo a partir de la caída del Muro de Berlín. Autores como Rancière (2007) y Badiou (2012) proponen que vivimos en un mundo donde existe un pacto entre los políticos y el capital. El Estado termina sirviendo más como un instrumento de la globalización para atraer capitales a los países que para garantizar el pacto social (Patiño-Patroni, 2012, p. 105). Estos cambios han traído como consecuencia que tengamos individuos que crecen alejados de la actividad política y dedicados a una vida de consumo (Bauman, 2007; Sennet, 2006). Portocarrero (2001) plantea que, como consecuencia de la implantación del modelo neoliberal, la subjetividad 
juvenil hegemónica cambió en el Perú. Desde la década de los noventa, la figura del hombre de éxito se ha impuesto sobre la del militante, la que era hegemónica antes de la caída del Muro de Berlín. Los jóvenes, siguiendo la tendencia global, se identifican más con un empresario emprendedor que con un activista político. La estabilidad de la economía y el fracaso de la izquierda como alternativa política permitieron que ese relato se mantuviera incólume hasta la segunda década del nuevo siglo. Al respecto, Golte plantea que los jóvenes peruanos son consumistas y polifacéticos debido a que «no hay reglas mínimas compartidas, ni hay metas generalizables para grupos mayores, salvo las que emanan de la imposición consumista» (2015, p. 33). No obstante, a pesar de que la subjetividad juvenil sigue ligada al consumo, en los últimos tiempos se han dado casos de participación política que rompen con el estigma de apatía política.

Un ejemplo de ello fue el de los ppkausas, los jóvenes que participaron en la campaña del mandatario peruano Pedro Pablo Kuczynski durante las elecciones generales del 2011 y del 2016. Dicho grupo llamó la atención porque estaba constituido por miembros de una franja etaria que no suele participar en carreras electorales y porque eligieron al candidato de mayor edad. Este grupo, siguiendo la tendencia de la época, se organizó a través de las redes sociales virtuales y, además, se expresó en las calles. A continuación, se indi- cará cuáles fueron las herramientas que se emplearon para poder analizar la red.

\section{Metodología: cartografiando a los ppkausas}

Para poder acercarse a los ppkausas, se decidió aplicar una estrategia cualitativa consistente en entrevistas a profundidad realizadas a 24 jóvenes que estuvieron presentes en los inicios de la creación del movimiento. Seguir esa estrategia permitió cumplir dos de los fines de este tipo de estudios: dar voz y hacer progresar la teoría (Ragin, 2007). Al darle voz a estos jóvenes, se logró un acercamiento exploratorio a las comunidades virtuales que se generan para desarrollar un activismo político. Asimismo, dicho enfoque permitió realizar un aporte a la teoría sobre las subjetividades juveniles, el activismo virtual y la participación política.

Esta investigación tiene como objetivo responder la pregunta principal de cuál fue la propuesta política de la red virtual formada por los seguidores del candidato Pedro Pablo Kuczynski en las elecciones generales peruanas del 2011. Dicha pregunta estuvo apoyada por las secundarias: cuáles fueron las subjetividades de los miembros del movimiento, qué similitudes y diferencias podemos encontrar con movimientos contemporáneos y, finalmente, de qué manera la red virtual expresaba las subjetividades de sus integrantes. Ante esas interrogantes, la hipótesis que se plantea es que la red 
cibernética formada por los seguidores de Pedro Pablo Kuczynski planteó una política conservadora que, a pesar de seguir las mismas estrategias de movimientos que apelan a la política insurgente, obedece a la subjetividad de sus miembros. Dicha subjetividad, vinculada al emprendedurismo y al conservadurismo, encontró en Kuczynski a la figura ideal para defender sus intereses individuales.

Para poder comprobar esta hipótesis, se eligió como herramienta de investigación la entrevista en profundidad. Dicha estrategia estuvo dirigida a ahondar en variables que, por un lado, permitieran reconocer la subjetividad de los primeros integrantes de la red juvenil y, por otro, ayudaran a reconocer el impacto que tuvieron las herramientas virtuales en la formación y expansión del movimiento. Por esa razón, las entrevistas estuvieron divididas en dos secciones. Las preguntas de la primera parte, presentadas para poder reconstruir una historia de vida de los entrevistados y así plantear una propuesta de subjetividad, estuvieron vinculadas a variables como edad, procedencia, nivel socioeconómico, y perspectivas laborales y familiares. Por su parte, las interrogantes de la segunda parte estuvieron dirigidas al ingreso de los consultados al movimiento, su participación en él y su evaluación de lo experimentado.

Para cumplir con ambos fines, se decidió hacer una selección de 24 jóvenes que es- que tuvieron una posición bastante cercana al candidato Kuczynski. Conversar con ellos permitió conocer por qué se apeló a las redes sociales, cómo se extendió el movimiento y cuáles fueron los discursos que se propagaron por la red. Las entrevistas, realizadas en un lapso de año y medio, empezaron un año después del fin de la campaña presidencial. Veintidós de ellas se llevaron a cabo en diferentes cafeterías y fuentes de soda de Lima, y las dos restantes en el que fuera el local de PPK ubicado en la Av. Salaverry. Todas las entrevistas se desarrollaron en un ambiente de cordialidad. Asimismo, el proceso de entrevistas también terminó asemejando la red que se había formado inicialmente: al fin de cada conversación, el consultado le brindó al entrevistador los datos de un contacto que había formado parte de los inicios del movimiento. De esa manera se pudo reconstruir la red constituida durante la campaña.

Dicha información sirvió para realizar una aproximación explorativa sobre las subjetividades que están en juego en los movimientos político-juveniles contemporáneos. Asimismo, el diseño empleado fue fenomenológico, porque se buscó, a través de las entrevistas, conocer la esencia del movimiento de los ppkausas. Esta información fue contrastada con la teoría proveniente de los Estudios Culturales presentada en el marco teórico, en aras de generar información relevante sobre las subjetividades y su relación con las redes sociales virtuales. 


\section{Una red cibernética}

Quizás la mejor frase para englobar lo que pasó en la campaña del 2011 es una de Caleb, uno de los miembros entrevistados de Generación Joven PPK: «lo que se dio fue una gran explosión de Facebook». Esas palabras recogen lo acontecido en los primeros meses de ese año, cuando el surgimiento de los ppkausas estuvo muy relacionado con las redes sociales virtuales. Tanto la prensa como los analistas políticos reportaron a inicios del 2011, casi con sorpresa, la estrategia de redes enfocada en los jóvenes que diseñó la campaña de Kuczynski (Barrenechea, 2011, p. 131). Dicha campaña fue diseñada por community managers y publicistas que querían resaltar los conocimientos tecnocráticos del candidato y que se inspiraron en la estrategia empleada por Barack Obama en el 2008. Se buscaba que PPK, al igual que el mandatario estadounidense, representara para los jóvenes la figura del cambio. Por esa razón, eligieron redes sociales como Facebook como el punto de partida.

Según lo relatado por Steven, otro miembro del colectivo, Generación Joven PPK se formó en julio del 2010 y justamente esa es la fecha de fundación que tiene un grupo de Facebook denominado Juventudes PPK. De acuerdo con Steven, el colectivo fue creciendo a medida que avanzaba la campaña y esta alza ocurrió gracias al trabajo realizado en redes sociales. Por lo tanto, lo que iremos viendo a lo largo de esta sección es que el movimiento ppkausa tuvo la estructura de una red social que se fue ampliando gracias a las convocatorias efectuadas en el ciberespacio. Por ejemplo, las declaraciones de la mayoría de los consultados indican que su ingreso a la campaña se produjo a través de esos espacios digitales. Las entrevistas permitieron recoger que muchos de ellos se enteraron de las propuestas del candidato gracias a los grupos creados en Facebook. En algunos casos, el paso natural era comentar en esos grupos y luego ser convocado a participar en las reuniones que se desarrollaban en la sede del movimiento. Por ejemplo, Sergio B. cuenta que empezó a comentar cuando «la página no llegaba ni a mil likes». De acuerdo con su testimonio, sus comentarios llamaron la atención de los administradores de la campaña, quienes decidieron convocarlo. Un caso similar es el de Alejandro, quien también fue invitado a participar por su presencia en las redes sociales. Steven mandó un correo electrónico cuya respuesta fue una invitación para hablar sobre la candidatura. Otros entrevistados, como Stephany, Jessica y Merly, se enteraron de las reuniones partidarias por Facebook y decidieron acercarse al local de la agrupación. Asimismo, un último grupo se unió gracias a la invitación de amigos que se habían integrado a través de las redes sociales. Por ejemplo, Leandro fue invitado por Steven, María del Pilar acompañó a Jessica y Juan José fue llamado por Luis, el coordinador de juventudes del movimiento. 
Luego, tras la adhesión al grupo, los ppkausas empezaron a hacer campaña a través de las redes sociales difundiendo información sobre las propuestas del candidato entre sus familiares y amigos. Miguel Ángel relata que dentro del colectivo se conversaba de las propuestas de PPK «para que al momento de salir a las calles se tuviera un mismo discurso». Caleb y Eliot también apuntan que los miembros de la red conversaban constantemente del plan de gobierno de Kuczynski para poder difundir esa información entre los electores. La mayoría de ellos señaló que gracias a la red accedió a los 25 puntos que constituían la base del plan de gobierno de la Alianza por el Gran Cambio, alianza política que lanzó la candidatura de PPK. Asimismo, los consultados también señalaron que dentro de la red se formaron vínculos que les permitieron sentirse parte de una comunidad. Por ejemplo, Leandro menciona que «se logró formar una comunidad, no solo en Lima, sino incluso en provincias». Stephany cuenta que fue «una experiencia muy bonita» porque los jóvenes pudieron trabajar en conjunto. Miguel también reconoce una comunidad, ya que afirma que «todos nos comprometimos en la causa de difundir el discurso del candidato». Sergio considera que al comienzo se formó «una comunidad netamente ppkausa enfocada en la candidatura presidencial». El objetivo de esa comunidad era contagiar su simpatía por el candidato. Eliot dice que buscaban que las personas que estaban fuera de la red se identificaran con sus ideales. Por su parte, Miguel es claro en señalar que se quería difundir el mensaje de Kuczynski y contagiar.

Lo relatado anteriormente muestra que el movimiento de los ppkausas cumple con los requisitos de una red social. Cada uno de ellos se acercó al colectivo porque se sintió atraído por el discurso de PPK, economista de profesión. Una vez dentro de la red, se constituyeron en nodos que estaban vinculados entre sí por el deseo de llevar a Kuczynski al poder. Asimismo, en la red circuló información sobre los planes del candidato que permitió que los vínculos se reforzaran. Una vez que recibieron la información, los integrantes del colectivo buscaron propagar las ideas de PPK para ampliar la red y conseguir electores obedeciendo al mecanismo del contagio. Por lo tanto, cada uno de estos jóvenes era un nodo que se dedicaba a difundir información sobre la campaña del candidato.

Lo que también es necesario reconocer es que la red de los ppkausas estuvo constituida por dos tipos de redes: una real' que se constituyó en las reuniones de campaña, y una digital, producto de los grupos de Facebook. Lo que hemos podido observar es que la segunda sirvió como instrumento de propagación de la primera debido a los beneficios que otorga lo digital. Las redes digitales siguen el que las redes digitales no son reales, ya que la experiencia dice lo contrario. 
modelo de las tradicionales (Christakis y Fowler, 2010), pero cuentan con una capacidad de expansión mucho mayor porque, como dependen de Internet, son el «medio de comunicación más rápido, autónomo, interactivo, reprogramable y autopropagable de la historia» (Castells, 2012, p. 32). Es por esa razón que movimientos sociales y políticos han encontrado en estas herramientas la posibilidad de ampliar su convocatoria y popularidad. Uno de los casos más célebres fue el del expresidente de EE. UU., Barack Obama, quien logró triunfar en las elecciones del 2008 gracias a su trabajo en esa plataforma (Castells, 2009). Este político empezó creando una red local para recaudar fondos para su campaña para luego transformarse en una figura conocida en todo el mundo y que tiene seguidores en diferentes países del globo. Justamente la campaña de Kuczynski vio en el caso del mandatario un ejemplo al cual imitar y buscó ser su réplica en el Perú. No es casualidad que se llamara inicialmente Generación Joven PPK, un émulo de la Generación Obama. Para fundamentar mi postura haré una comparación entre ambos casos que más adelante me servirá para demostrar que la red de los ppkausas tenía un objetivo distinto a la de la política insurgente de Obama.

\section{La propuesta conservadora de los pplausas}

Si bien la campaña de PPK realizó una estrategia similar a la de Obama, podemos encontrar importantes diferencias entre una y otra. Propongo que, a pesar de que la palabra cambio fue usada por ambas campañas, tuvo un significado distinto en cada caso. Castells (2009) plantea el caso del mandatario estadounidense como un ejemplo de política insurgente porque logró conectar «a los segmentos de gente sin poder con los procedimientos de ejercicio del poder» (2009, p. 475). Casi una década después de su llegada a la presidencia, son pocos los que se atreverían a decir que Obama es un referente de política insurgente, debido a que las acciones que llevó a cabo durante su Gobierno favorecieron a los grupos de poder económico de su país. Sin embargo, no se puede negar que, durante la campaña que realizó en el 2008 para acceder a la candidatura demócrata, el mandatario planteó acciones que representaron una alternativa a la política tradicional. El entusiasmo que generó en activistas de EE. UU. y del mundo se debió a que creó la conciencia de que podía existir una alternativa a la política dirigida por las grandes corporaciones y marcó el camino para movimientos como Occupy Wall Street (OWS) o la campaña del precandidato demócrata Bernie Sanders.

No es posible decir lo mismo de la campaña de PPK. Anteriormente, propuse que los ppkausas obedecen a la subjetividad del militante del éxito. Dicha subjetividad constituye una paradoja, ya que, a pesar de que se adhiere a una revolución - la capitalista-, propone una 
militancia conservadora. Los ppkausas son conservadores porque defienden el camino trazado por el neoliberalismo, pero revolucionarios porque se plantean reformar todo lo que obstaculice ese modelo. Por lo tanto, el significante cambio tenía un sentido distinto. Su propuesta estaba emparentada con reformas que permitían que el sistema neoliberal planteado por los Gobiernos peruanos desde la década de los noventa termine de establecerse. Una de ellas sería la reforma de la política. Por ejemplo, en varios anuncios publicados en Facebook aparecían mensajes en los cuales Kuczynski llamaba a sus seguidores a reformar la política. Mensajes como «Jóvenes no dejen que la política los forme, formen ustedes la nueva política»o «La esperanza está en los jóvenes que aún creen en la política como servicio» se encontraban publicados en las redes sociales ${ }^{3}$. Como se pudo comprobar en las entrevistas, los ppkausas coincidían con ese mensaje debido a que indicaban que los políticos son personajes que solo se benefician de sus cargos y varios de ellos consideraban que debían ser reemplazados por personas capaces o técnicos. Por lo tanto, el gran cambio al que eran llamados a participar los ppkausas estaba relacionado con la renovación de los políticos que obstaculizan el desarrollo del país y no llegó a ser una propuesta de insurgencia como la de Obama.
Asimismo, el sentido de cambio de la red de PPK también se explica por otros integrantes que formaron parte del colectivo. Castells indica que las redes no están compuestas por «actores aislados (individuos, grupos, clases, líderes religiosos o políti$\cos$ )», sino que «requiere de un complejo grupo de acción conjunta que trasciende las alianzas hasta convertirse en una nueva forma de sujeto» (2004, p. 61). Los ppkausas solo fueron una parte de una compleja red con tendencias conservadoras. En la elección del 2011, el economista fue apoyado, primero, por una alianza de partidos políticos de diferentes tendencias que han estado relacionadas con el poder nacional o regional en varias oportunidades y, segundo, por una serie de personajes ligados al empresariado nacional.

Por lo tanto, a pesar de que ambas redes manejaron estrategias de contagio similares, sus propuestas eran distintas. Mientras que la de Obama nació con el propósito de realizar una política insurgente ajena a los órganos que administran el poder, la de PPK se constituyó desde una propuesta conservadora en la cual los grupos de poder político y económico tenían un papel importante. Este hecho provoca que sean redes con diferentes propósitos que solo tendrían en común el haberse estructurado sobre la base del significante cambio. En la siguiente sección, compararé a los ppkausas con otros

${ }^{3}$ Dichos mensajes se encontraban en la página de Facebook Generación Joven PPK. Actualmente, el nombre ha cambiado a Juventudes PPK (https://www.facebook.com/pages/Generación-Joven-PPK/132615773450055?fref=ts). 
grupos contemporáneos que plantean una forma de hacer política alejada de las élites. Esa comparación servirá para fundamentar que este movimiento no se adhiere a las propuestas contemporáneas que plantean un nuevo espacio público formado por ciudadanos.

\section{Indignados y okupas}

Como se indicó anteriormente, desde el 2011, en diferentes lugares del planeta han ido apareciendo movimientos sociales que han puesto en cuestión el orden político y económico de sus países. Desde el Medio Oriente, pasando por Europa, Estados Unidos y llegando a América Latina, se han podido ver casos de movimientos que se generaron en las redes sociales y que luego tomaron el espacio público (Alaminos y Penalva-Berdú, 2016; Badiou, 2012; Castells, 2012; Rodríguez, 2013). Estos grupos asumieron una actitud insurgente al construir espacios autónomos en lugares emblemáticos de las ciudades que asemejaban a las «barricadas» que levantaban los revolucionarios franceses (Castells, 2012). Dichas barricadas se planteaban como una reacción motivada por la indignación que generan las desigualdades producidas por un sistema dominado por los grandes capitales mundiales. Desde esos espacios se busca ejercer un contrapoder, «el intento deliberado de cambiar las relaciones de poder» (Castells, 2012, p. 26). Es por ello que podemos indicar que plantean una política insurgente.
De acuerdo con Castells, el ejercicio del contrapoder requiere del surgimiento de movimientos sociales que se constituyan a partir de «un proceso de comunicación autónoma, libre del control institucional» (2012, p. 27). Es allí donde son claves redes sociales digitales, como Facebook, Twitter o Tuenti. Movimientos como los indignados de España, la Primavera Árabe, Occupy Wall Street o \#YoSoy132 usaron estas nuevas herramientas para no verse influidos por los órganos de poder institucionales y generar su propia organización y agenda. Si antes los partidos políticos podían marcar la pauta a partir de la dominación de los medios de comunicación tradicionales, las redes sociales virtuales, al ser medios de autocomunicación de masas, resultan más difíciles de controlar. Allí los movimientos sociales lograron generar sus propias redes y plantar resistencia a los órganos de poder a partir de acciones de desobediencia civil como la toma de lugares públicos con gran importancia simbólica. Asimismo, dichos movimientos se caracterizaron por tener una fuerte presencia juvenil (Rodríguez, 2013). Aparentemente, los jóvenes habían encontrado las herramientas adecuadas para evitar verse contaminados por las formas tradicionales de dominación de las masas. Las redes sociales juveniles se plantean como el contrapoder que se opone al orden establecido.

Es en ese contexto donde se ubicaron los ppkausas. Hemos visto que la indignación fue la emoción que unió a los nodos 
de las redes virtuales de los movimientos sociales contemporáneos. En principio, se puede reconocer un sentimiento similar; sin embargo, se podrá observar que la indignación está dirigida a un solo grupo vinculado al poder. Por ejemplo, María del Pilar, miembro del colectivo Generación Joven PPK, señala que los políticos solo buscan su conveniencia. Por su parte, Jessica considera que la política está llena de candidatos «que prometen y no cumplen». Sergio B. opina que en los partidos políticos hay «cero institucionalidad». Caleb considera que la política no es usada para aportar a la sociedad, sino para aprovecharse de ella. Se puede leer en sus declaraciones rechazo y desaprobación hacia la clase política. Su participación en campaña se debió al deseo de cambiar esa situación y de reprogramar la forma de hacer política en el país. Steven señaló que decidió integrarse «a un grupo joven y nuevo con ideas de cambio y una nueva forma de hacer política que sea más horizontal». Gabriela se unió al movimiento porque le «interesa ser partícipe de nuevos cambios» que solo los jóvenes pueden lograr. Bajo esos deseos se construyó la primera estructura horizontal que se extendió por las redes sociales de forma rápida y autónoma. Una indignación contra el statu quo que se volcó en el deseo de colocar a PPK en el poder. Sin embargo, dicha indignación solo estaba dirigida hacia una parte de la élite del poder.

Desde la década de los noventa, el Perú ha clase política y los empresarios que busca mantener en el país un modelo neoliberal que debilita la participación ciudadana (Cameron, 2009; Durand, 2007; Vergara, 2012). Por lo tanto, la política -los aparatos políticos que constituyen el Estadoen el Perú está integrada por políticos y empresarios. Como hemos podido observar, la red de los ppkausas solo rechazaba una parte de la política: los políticos. Y no a todos los políticos, solo a aquellos que aparecían como un peligro para el progreso. Asimismo, dicho sentimiento no incluía a los empresarios. Por el contrario, como se ha indicado anteriormente, la red estaba fuertemente conectada con los intereses de los que ejercen el poder económico. Tanto los creadores de la red como los miembros de la agrupación política formaban parte de esos sectores y terminaron evitando que esté alejada de los programadores que ostentan el poder en el Perú. En esas circunstancias, dar el segundo paso - tomar el espacio público, establecer la barricada- era indeseable.

Recordemos que los ppkausas son jóvenes conservadores que buscan que la revolución capitalista siga extendiéndose. Por lo tanto, sus objetivos se alejan de la toma del espacio público, de crear un espacio autónomo a costa de los centros de poder político y económico. Tomemos en cuenta que grupos como OWS y los indignados del 15-M español realizaron sus acampadas en sitios emblemáticos, lugares que representaban a los órganos de poder y al flujo del dinero. No es ca- 
sualidad que OWS haya decidido tomar un parque que se encontraba muy cerca de Wall Street, el lugar que simboliza el movimiento de las finanzas mundiales. Ese tipo de insurgencia pretende detener el flujo de dinero mundial, paralizar los procesos de la globalización. Esa idea se aleja de una subjetividad como la de los ppkausas, muy vinculada a la sociedad de consumo global.

Aquí podemos acudir a algunos autores asociados a los Estudios Culturales para reconocer las subjetividades de los actores que encabezaron la red ppkausa. Siguiendo las ideas de Castells (2012), podemos entender por qué ellos no querían formar un sitio autónomo: no eran partidarios de una revolución, solo de reformas que no afecten lo que ellos consideraban una revolución capitalista. De acuerdo con sus discursos, su objetivo era retirar los obstáculos del espacio existente para que siga avanzando el crecimiento económico que ofrece el sistema neoliberal. Las movilizaciones debían servir para golpear a los políticos que amenazaban el movimiento del capital. Recordemos que Rancière (2007) y Badiou (2012) proponen que vivimos en un mundo donde existe un pacto entre los políticos y el capital. Los indignados de varios países del mundo cuestionan ese pacto. Pero no era el caso de los partidarios de Kuczynski. Los ppkausas son jóvenes conformes con el sistema económico. Por ejemplo, para Manuel y Gabriel solo hay que realizar ajustes; para María del Pilar y Alejandro el país está en buen camino. La mayoría de estos jóvenes piensa que solo es necesario plantear reformas en áreas como la educación y cambiar a los políticos.

Por lo tanto, dichos discursos pondrían en evidencia que la subjetividad de los integrantes de la red ppkausa se articula con el discurso hegemónico existente en el Perú desde la década de los noventa. La red habría funcionado como un eje articulador de un grupo de nodos que promovían la permanencia del sistema neoliberal, porque encontraban en el partido de Kuczynski un espacio acorde a sus subjetividades. Esa mirada se conectaría con la propuesta de Han (2014) sobre las redes como un enjambre digital formado por individuos aislados que siguen la tendencia neoliberal de la época. Los ppkausas se habrían reunido en esta red porque habrían visto en ella la posibilidad de crecimiento económico que promete el sistema neoliberal. Asimismo, la subjetividad de los miembros de la red también se conecta con las propuestas de Bauman (2007) y Sennet (2006). Una vez concluida la elección, los integrantes de la red consultados se separaron de esta. En las entrevistas, indicaron que debían continuar con sus carreras universitarias, el paso previo al éxito profesional, y por ello dejaban de tener tiempo para el activismo político. Lo que se desprende de dichas afirmaciones es que su compromiso con la red era débil. Los ppkausas entrevistados evidencian la tendencia de la sociedad de consumo, una en la que 
el sujeto no se compromete con sus relaciones. La tendencia consumista de descartar los objetos se replica en la acción política. Por ello, la red se desmembró cuando acabó la elección. Sus miembros sintieron que la tarea había concluido al finalizar la campaña.

En consecuencia, a la luz de la comparación con los movimientos sociales, se podría decir que el espacio de los ppkausas no buscó ser autónomo. Su espacio habría estado más vinculado a lo doméstico, a lo conservador y no a lo diferente. Preferirían la ppkasa que propone la revolución capitalista y no la barricada de la política insurgente.

\section{A manera de conclusión: la defensa de la ppkasa}

Hemos visto que el surgimiento durante los últimos años de movimientos sociales que utilizan las redes sociales virtuales para organizarse ha provocado que estas herramientas tecnológicas sean consideradas como una alternativa a la programación planteada por los medios de comunicación masiva tradicionales. Estos colectivos habrían logrado generar espacios autónomos alejados de la influencia del poder oficial y aplicar una política insurgente gracias a las redes mencionadas. Sin embargo, en este artículo se ha podido analizar el caso de un grupo nacido en la virtualidad que trabajó a partir de la agenda de los órganos que es posible detectar que dichas redes también podrían ser tomadas por los centros que ejercen el poder. En ese sentido, lo que se concluye es que las redes sociales virtuales no solo servirían para generar espacios autónomos, sino que podrían funcionar como caja de resonancia de los grupos conservadores.

El caso de los ppkausas demostraría que las redes sociales pueden operar como enjambres digitales formados por sujetos individualistas cuya indignación no esté dirigida a buscar que el statu quo cambie. Dichos enjambres tendrían como función agrupar a individuos que piensan más en el beneficio personal que en el colectivo. Son sujetos que obedecen al espíritu de la época, uno que indica que la felicidad está vinculada al consumo o el emprendimiento. Asimismo, la red se desmembró al finalizar la elección. Sus participantes consideraron que no era necesario seguir en el activismo político una vez concluida la campaña. Dichas acciones también se podrían vincular a la tendencia actual a evadir el compromiso, en este caso político, propia de la sociedad de consumo. En suma, el activismo político de los ppkausas tenía fecha de caducidad.

En consecuencia, podríamos identificar que la propuesta política de la red virtual formada por los seguidores del candidato Pedro Pablo Kuczynski en las elecciones del 2011 habría sido conservadora porque buscaba defender el sistema neoliberal y obedecía a las dinámicas de la sociedad 
de consumo. Dicha estructura les habría servido para ponerse en contacto con los grupos de poder que defienden el estilo de vida al que aspiran. Por lo tanto, la red no habría representado una barricada que entorpeciera el sistema; por el contrario, se habría ofrecido como un espacio para reunir a los que buscaban defenderlo. No habría sido una apuesta por lo nuevo, sino por lo conocido. Los seguidores de Kuczynski no se habrían atrincherado en las barricadas: habrían apostado por la ppkasa.

\section{REFERENCIAS}

Alaminos-Chica, A. y Penalva-Verdú, C. (2016). Visions of the Spanish Revolution: Identities and conflicts in post-welfare societies. Convergencia. Revista de Ciencias Sociales, 23(70), 13-33.

Badiou, A. (2012). El despertar de la historia. Madrid, España: Clave Intelectual.

Bauman, Z. (2007). Vida líquida. Madrid, España: Paidós.

Barrenechea, R. (2011). PPK: auge y caída de una ilusión electoral. En C. Meléndez (Comp.), Post candidatos. Guía analítica de supervivencia hasta las próximas elecciones (pp. 123-145). Lima, Perú: Aerolíneas Editoriales.

Cameron, M. (2009). El giro a la izquierda frustrado: el caso de Ollanta Humala. Convergencia. Revista de Ciencias Sociales, 16(Núm. Esp. IA), 275-302.

Castells, M. (Ed.). (2004). La sociedad red: una visión global. Madrid, España: Alianza Editorial.

Castells, M. (2009). Comunicación y poder (Trad. M. Hernández). Madrid, España: Alianza Editorial.

Castells, M. (2012). Redes de indignación y esperanza (Trad. M. Hernández). Madrid, España: Alianza Editorial.

Christakis, N. y Fowler J. (2010). Conectados. El sorprendente poder de las redes sociales y cómo nos afectan. Madrid, España: Taurus.

Durand, F. (2007). El Perú fracturado. Formalidad, informalidad y economía delictiva. Lima, Perú: Fondo Editorial del Congreso del Perú.

Golte, J. (2015). Análisis de la situación política, social y económica de los jóvenes en el Perú. En E. Rodríguez y J. Corcuera (Eds.), Subjetividades diversas. 
Análisis de la situación política, social y económica de las juventudes peruanas (pp. 25-34). Lima, Perú: Secretaría Nacional de la Juventud.

Han, B. (2014). En el enjambre. Barcelona, España: Herder Editorial.

León, D. (2013). Diversión y protesta mediatizadas: características y alcances sociales de los flashmobs en Lima. En E. Rodríguez (Ed.), Movimientos juveniles en América Latina y el Caribe: entre la tradición y la innovación. (pp. 249-265). Lima, Perú: Secretaría Nacional de la Juventud.

Patiño-Patroni, A. (2012). Nombrar la nación: el inconsciente político de la publicidad contemporánea en el Perú. Recuperado de https://www.academia.edu/2314265/Nombrar_la_Nación_El_inconciente_pol\%C $3 \% \mathrm{AD}$ tico_de_la_publicidad_contemporánea_en_el_Perú

Portocarrero, G. (2001). Nuevos modelos de identidad en la sociedad peruana (hacia una cartografía de los sentidos comunes emergentes). En G. Portocarrero y J. Komadina (Eds.), Modelos de identidad y sentidos de pertenencia en Perú y Bolivia, (pp. 11-84). Lima, Perú: Instituto de Estudios Peruanos.

Ragin, C. (2007). La construcción de la investigación social. Introducción a los métodos y su diversidad. Bogotá, Colombia: Siglo del Hombre Editores.

Rancière, J. (2007). El odio a la democracia. Buenos Aires, Argentina: Amorrortu.

Rivas, B. (2014). Ppkausas, defensores del nuevo capitalismo peruano. Un estudio del discurso de 24 jóvenes que participaron en la campaña de Pedro Pablo Kuczynski (Tesis de maestría, Pontificia Universidad Católica del Perú, Lima, Perú), Recuperada de http://tesis.pucp.edu.pe/repositorio/
Rodríguez, E. (2013). Movimientos juveniles en América Latina: entre la tradición y la innovación. En E. Rodríguez (Ed.), Movimientos juveniles en América Latina y el Caribe: entre la tradición y la innovación (pp. 19-34). Lima, Perú: Secretaría Nacional de la Juventud.

Sennet, R. (2006). La cultura del nuevo capitalismo (Trad. M. A. Galmarini). Barcelona, España: Anagrama.

Venturo, S. (2001). Contrajuventud. Ensayos sobre juventud y participación política. Lima, Perú: Instituto de Estudios Peruanos.

Vergara, A. (julio, 2012). Alternancia sin alternativa: ¿un año de Humala o veinte años de un sistema? Argumentos, (3). Recuperado de http://revistaargumentos.iep.org.pe/articulos/alternancia-sin-alternativa-un-ano-de-humala-o-veinte-anos-de-un-sistema/ 